\title{
Spontaneous Termination of Atrial Fibrillation: A Challenge from PhysioNet and Computers in Cardiology 2004
}

\author{
GB Moody
}

Harvard-MIT Division of Health Sciences and Technology, Cambridge, MA, USA

\begin{abstract}
The fifth annual PhysioNet/Computers in Cardiology Challenge asked if it is possible to predict if (or when) an episode of atrial fibrillation (AF) will end spontaneously. We prepared a set of $A F$ recordings including examples of both sustained and spontaneously terminating AF; transitions to sinus rhythm were not shown in these examples. We posted the recordings on PhysioNet, and invited participants to classify them. Over twenty teams participated, and most were able to distinguish between sustained and spontaneously terminating AF with high accuracy.
\end{abstract}

\section{Introduction}

Several years ago, the second PhysioNet/Computers in Cardiology Challenge posed the problem of predicting the onset of paroxysmal atrial fibrillation [1]. This challenge returns to the subject of $\mathrm{AF}$, focussing on predicting if and when an episode of AF will self-terminate. Unlike venticular fibrillation, which is invariably fatal if it is not interrupted, it is possible for atrial fibrillation to be sustained indefinitely, since the ventricles continue to perform the essential function of driving the circulation, albeit inefficiently. The risks of sustained atrial fibrillation are nevertheless serious, and include strokes and myocardial infarctions caused by the formation of blood clots within stagnant volumes in the atria. Evidence suggests that spontaneously terminating (paroxysmal) atrial fibrillation, or PAF, is a precursor to the development of sustained AF.

Although spontaneously terminating episodes of AF are often very short (perhaps a few seconds in duration), it is interesting to note that longer episodes lasting several minutes also occur. These appear to be very similar to sustained (non-terminating) AF. Subtle changes in rhythm during the final minutes or seconds of such episodes may lead to (or predict) termination of AF. Improved understanding of the mechanisms of spontaneous termination of atrial fibrillation may lead to improvements in treatment of sustained AF. If it were possible to recognize the conditions under which PAF is likely to self-terminate, it might also be possible to intervene in affected individuals to increase the likelihood of self-termination of what would otherwise be sustained AF.

\section{Methods}

The challenge required a collection of AF recordings of known types. We reviewed RR interval scattergrams (see figure 1) and instantaneous heart rate tachograms from a large set of long-term (20-24 hour) ECG recordings, in order to locate episodes of sustained AF (defined as episodes of durations exceeding one hour that did not terminate before the end of the recording), and episodes of PAF that lasted at least one minute (in many cases, at least two minutes). We then examined the ECG signals at high resolution in areas of interest identified from the scattergrams, and selected sustained AF and PAF excerpts to be used. In all, we included 80 one-minute recordings of AF from 60 different subjects in the AF Termination Challenge Database, and posted them on PhysioNet [2].

Each record in the database is a one-minute segment of atrial fibrillation, containing two ECG signals, each sampled at 128 samples per second, accompanied by a set of QRS annotations produced by an automated detector, in which all detected beats, including any ectopic beats, are labelled as normal. These annotations have not been audited and may contain a small number of errors.

The database is divided into a learning set (records with names of the form $\mathrm{n}^{*}, \mathrm{~s}^{*}$, and $\mathrm{t}^{*}$ ) and two test sets (records with names of the form $\mathrm{a}^{*}$ and $\mathrm{b}^{*}$ ). The learning set contains 30 records in all, with 10 records in each of three groups (see figure 2 for examples):

- Group $N$ (records n01, n02, .. n10): non-terminating AF (defined as AF that was not observed to have terminated for the duration of the long-term recording, at least an hour following the segment).

- Group $S$ (records s01, s02, ... s10) AF that terminates one minute after the end of the record.

- Group T (records t01, t02, ... t10) AF that terminates immediately (within one second) after the end of the record. Note that these records come from the same long-term ECG recordings as those in group $\mathrm{S}$ and immediately fol- 


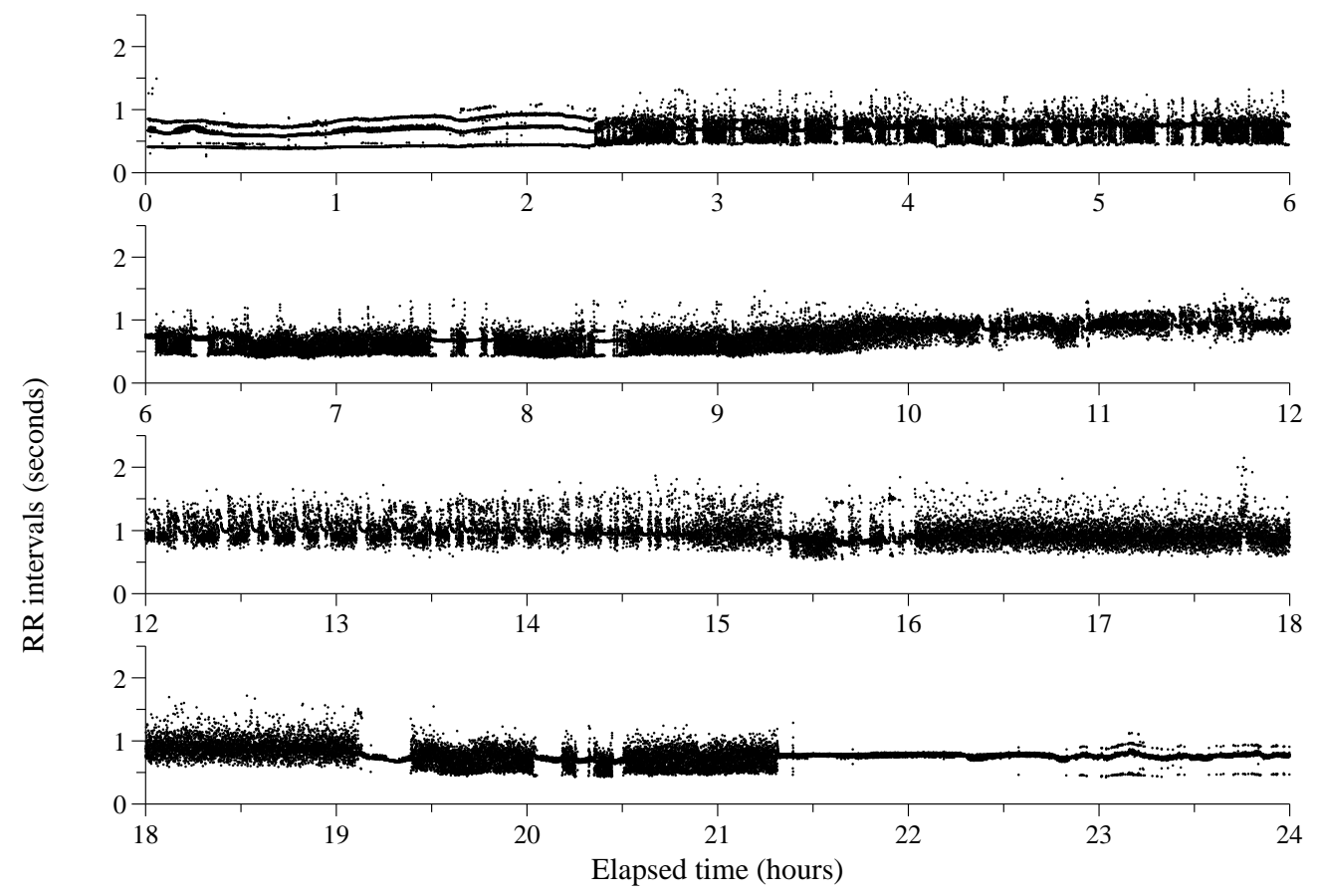

Figure 1. A typical 24-hour RR interval scattergram. Each trace shows all measured RR intervals during a six-hour period. A variety of rhythms can be identified in this example, including ventricular bigeminy (top trace, left), normal sinus rhythm (e.g., hour 22), and numerous episodes of AF of durations ranging from a few beats (e.g., hour 14) to several hours (hours 16-19).

low the Group S records (for example, t01 is the continuation of s01).

The learning set records were obtained from 20 different subjects (10 group $\mathrm{N}, 10$ group $\mathrm{S} / \mathrm{T}$ ).

Test set A contains 30 records $(\mathrm{a} 01, \mathrm{a} 02, \ldots \mathrm{a} 30)$ from 30 subjects (none represented in the learning set or in test set B). Approximately half of these records belong to group $\mathrm{N}$, and the others belong to group $\mathrm{T}$. The goal of the first challenge event is to identify which records in test set A belong to group $\mathrm{T}$.

Test set B contains 20 records (b01, b02, ... b20), 2 from each of 10 subjects (none represented in the learning set or in test set A). One record of each pair belongs to group S, and the other to group T; note, however, that there are short gaps (of less than one second) between some of these pairs. The goal of the second challenge event is to identify which records belong to group $\mathrm{T}$.

Participants submitted their classifications of the test set records via a web form to an autoscorer that we provided on PhysioNet, and received their results (aggregate scores for each event, with one point for each correctly classified record) by return email. Each team was allowed up to five attempts in each event, and only the best score received in each event was used to determine the final rankings.

\section{Results}

Over twenty teams participated in the Challenge. Most were able to develop methods for discriminating with reasonable accuracy between PAF and sustained AF (event 1), with six groups classifying $90 \%$ or more of the test set A records correctly (see table 1). 
Table 1. Final results for event 1 (sustained vs. self-terminating AF).

\begin{tabular}{|l|l|}
\hline Score & Entrant \\
\hline $29(97 \%)$ & $\begin{array}{l}\text { S Petrutiu, AV Sahakian, J Ng, S Swiryn } \\
\text { Northwestern University, Evanston, Illinois, USA }\end{array}$ \\
\hline $28(93 \%)$ & $\begin{array}{l}\text { D Hayn, K Edegger, D Scherr, P Lercher, B Rotman, W Klein, G Schreier } \\
\text { ARC Seibersdorf Research GmbH; Medical University of Graz, Austria }\end{array}$ \\
\hline $27(90 \%)$ & $\begin{array}{l}\text { F Cantini, F Conforti, M Varanini, F Chiarugi, G Vrouchos } \\
\text { CNR Institute of Clinical Physiology, Pisa, Italy; ICS-FORTH, Heraklion, Greece } \\
\text { M Lemay, Z Ihara, JM Vesin, L Kappenberger } \\
\text { EPFL - CHUV, Lausanne, Switzerland }\end{array}$ \\
$\begin{array}{l}\text { F Castells, C Mora, R Ruiz, JJ Rieta, J Millet, C Sanchez, S Morell } \\
\text { Universidad Politécnica de Valencia; Hospital Clinico Universitario de Valencia; Universidaa de } \\
\text { Castilla la Mancha, Cuenca, Spain }\end{array}$ \\
$\begin{array}{l}\text { F Nilsson, M Stridh, A Bollman, L Sornmö } \\
\text { Lund University, Sweden; Good Samaritan Hospital and Harbor-UCLA Medical Center, Los Ange- } \\
\text { les, California, USA }\end{array}$ \\
\hline
\end{tabular}

Most participants also attempted to classify test set B into AF episodes that terminate immediately and other episodes that terminate one minute after the end of the excerpt (event 2). Only three teams achieved results of $90 \%$ or better in this event (see table 2).

\section{Discussion and conclusions}

Several of the most successful approaches to event 1 began by subtracting the QRS complexes from the ECG signals, followed by frequency-domain analysis of the residual signals, which were dominated by atrial activity. Evidence gathered from study of the learning set supported the hypothesis that atrial activity slows and regularizes prior to self-termination of AF.

Many teams found event 2 to be significantly more difficult than discriminating between PAF and sustained AF. A few teams exploited the structure of test set B, and began by identifying which pair of records appeared to have come from each subject. Once the records had been sorted in this way, these participants looked for the record in each pair that appeared to be most similar to the PAF examples in the learning set and in test set A.

The major finding is that PAF can be distinguished accurately from sustained AF by analysis of a minute or less of the ECG. Recognition of the conditions under which AF self-terminates is a first step toward the development of therapeutic interventions that may guide the state of individuals experiencing sustained AF towards self-terminating AF.

The database will remain available for further study at http://www.physionet.org/physiobank/database/aftdb/.

\section{Acknowledgements}

PhysioNet/Computers in Cardiology Challenges are conducted using the facilities of PhysioNet, a public service of the Research Resource for Complex Physiologic Signals, which is supported by a grant from the National Center for Research Resources of the US National Institutes of Health (P41 RR13622). The author and his colleagues at PhysioNet are grateful to Steven Swiryn, of Northwestern University, for proposing the challenge topic, and for providing and reviewing a large set of ambulatory ECG recordings from which the data for the challenge were selected. The author also wishes to thank Julia Hand of Northwestern, and Isaac Henry of the Beth Israel Deaconess Medical Center and PhysioNet, for invaluable assistance in data preparation; and the Board of Computers in Cardiology for their sustained and wholehearted support of these challenges. Finally, the author thanks all of the participants in this year's PhysioNet/Computers in Cardiology Challenge, many of whom report their results elsewhere in this volume.

\section{References}

[1] Moody GB, Goldberger AL, McClennen S, Swiryn S. Predicting the onset of paroxysmal atrial fibrillation: The Computers in Cardiology Challenge 2001. Computers in Cardiology 2001;28:113-116.

[2] Moody GB, Mark RG, Goldberger AL. PhysioNet: A research resource for studies of complex physiologic and biomedical signals. Computers in Cardiology 2000;179-182. 
Table 2. Final results for event 2 ( $\mathrm{AF}$ terminating in one minute vs. immediately).

\begin{tabular}{|l|l|}
\hline Score & Entrant \\
\hline $20(100 \%)$ & $\begin{array}{l}\text { S Petrutiu, AV Sahakian, J Ng, S Swiryn } \\
\text { Northwestern University, Evanston, Illinois, USA }\end{array}$ \\
\hline $18(90 \%)$ & $\begin{array}{l}\text { F Cantini, F Conforti, M Varanini, F Chiarugi, G Vrouchos } \\
\text { CNR Institute of Clinical Physiology, Pisa, Italy; ICS-FORTH, Heraklion, Greece } \\
\text { B Logan, J Healey } \\
\text { Hewlett Packard Laboratories, Cambridge, MA, USA }\end{array}$ \\
\hline $16(80 \%)$ & $\begin{array}{l}\text { Q Xi, S Shkurovich } \\
\text { St. Jude Medical, Sylmar, CA, USA }\end{array}$ \\
& $\begin{array}{l}\text { D Hayn, K Edegger, D Scherr, P Lercher, B Rotman, Klein, G Schreier } \\
\text { ARC Seibersdorf Research GmbH; Medical University of Graz, Austria }\end{array}$ \\
\hline
\end{tabular}

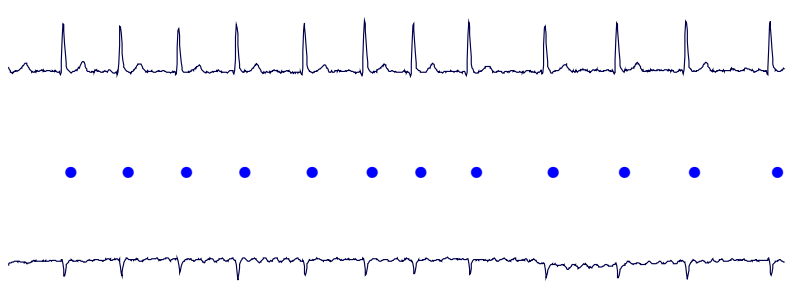

Address for correspondence:

George B. Moody

MIT Room E25-505A, Cambridge, MA 02139 USA

george@mit.edu
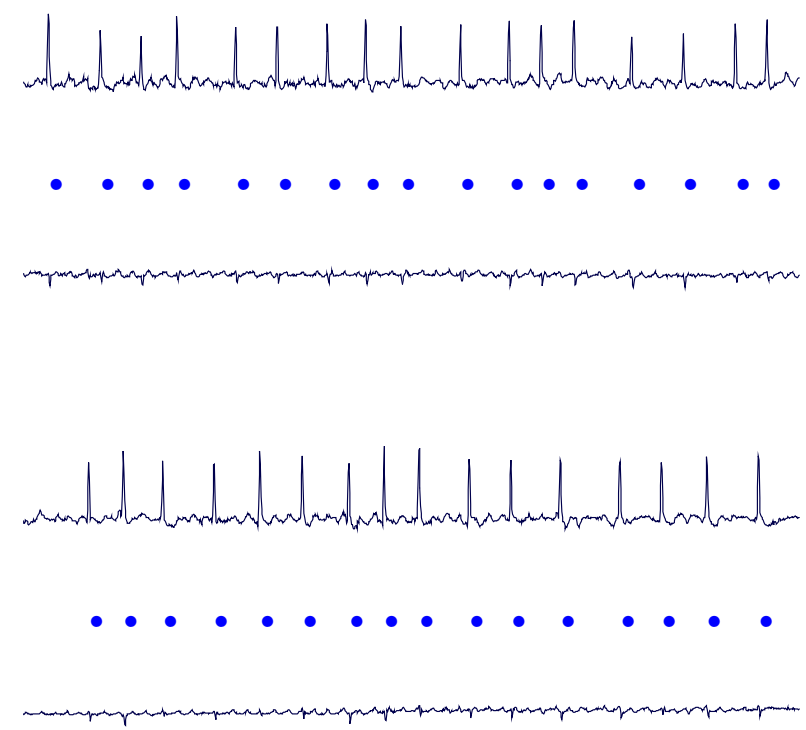

Figure 2. Examples from the AF Termination Challenge Database. The three panels show the final 10 seconds of each of records n02 (top), s02 (center), and t02 (bottom). Annotations between the two ECG signals from each record mark the locations of the QRS complexes. Contrast the atrial activity in the upper panel (sustained AF) with that in the other two panels (PAF). 